\title{
ULYSSES JUPITER FLY-BY A Wealth of New Results
}

The primary goal of the Ulysses Jupiter fly-by of 31 January to 16 February, 1992, was to place the spacecraft in its final heliocentric out-of-ecliptic orbit with a minimum of risk to the onboard systems and scientific payload. Scientific investigations in the Jovian magnetosphere were a secondary objective. Furthermore, many of the instruments are optimised for the conditions encountered in the solar wind, including their orientation on the spinning spacecraft. In spite of these constraints, the Ulysses experiments have produced a wealth of new information about the fascinating Jovian magnetosphere.

Two factors contributed to this success. Firstly, the fly-by trajectory (see figure), leaving the Jovian system via the previously unexplored dusk sector of the magnetosphere took Ulysses to higher latitudes than Pioneer and Voyager spacecraft. Secondly, the 11 instruments carried by Ulysses are in many cases more powerful than on the previous missions. Noteworthy features were continuous coverage in frequency from 0 to $1 \mathrm{MHz}$ provided by the magnetometer and radio/plasma wave experiments and continuous coverage in charged-particle energies (see page 175) from a few hundred $\mathrm{eV}$ (solar wind electrons) to hundreds of $\mathrm{MeV}$ (primary cosmic rays).

Almost a week before closest approach, Ulysses crossed the Jovian bow shock at a distance of 113 Jupiter radii $\left(R_{\mathrm{J}}\right)$ from the planet. The inbound crossing occurred somewhat earlier than expected based on previous observations. A possible interpretation is that the magnetosphere was temporarily "inflated" by hot plasma flowing away from the planet. The magnetopause was encountered only four hours after the bow shock crossing, at around $110 R_{\mathrm{J}}$. The apparent proximity of these two boundaries suggests that they were moving rapidly outwards at that time.

Ulysses has provided important new data on the composition of these magnetospheric plasma, including charge-state information. These measurements will provide new insights into the sources and "life history" of the Jovian plasma. For example, sulfur and oxygen ions, originating from the satellite lo, were detected in the outer magnetosphere, at large radial distances from the planet, for the first time. The lo plasma torus was an object of special interest during the fly-by. Measurements taken a few hours after closest approach as the spacecraft crossed Jupiter's magnetic equator, indicate that the electron density of the torus was quite similar to predictions based on the Voyager results. On the other hand, the longitudinal distribution of plasma showed unexpected asymmetries. These features were observed both directly by the plasma wave instruments, and also remotely by the radio science team.

Because of its high-latitude trajectory, Ulysses was able to investigate the poleward extent of the Jovian radiation belts that contain durably trapped energetic electrons

A shematic illustration of the Jovian magnetosphere. Jupiter is a strongly magnetized, rapidly rotating planet with the largest magnetosphere in the solar system. The large Galilean satellites are embedded within the magnetosphere and the moon lo is known to be a prolific source of ions and particles. Ions, predominantly oxygen and sulphur, form a large torus a doughnut-shaped ring of plasma rotating with the planet at the 10 orbit. Electrons from 1o, Jupiter's

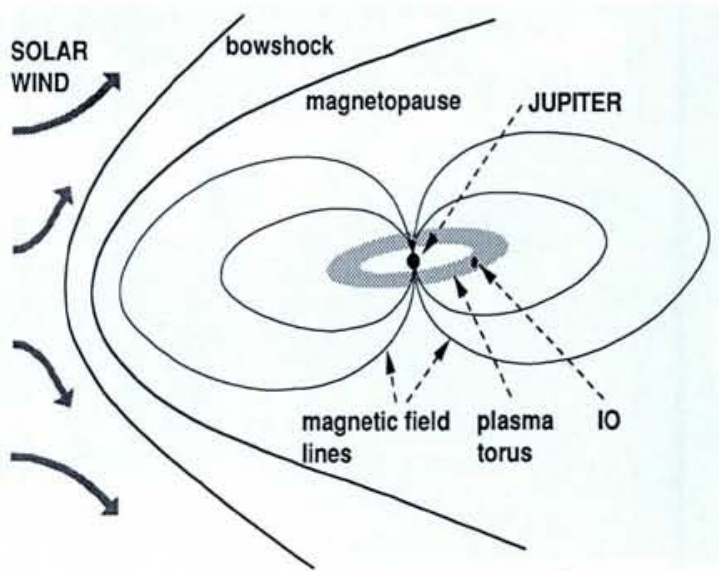
ionosphere, and the solar wind are all present and are transported throughout the magnetosphere. Many of the particles are accelerated to extremely high energies to form intense radiation belts. The presence of the ions in the rapidly rotating magnetic field causes the lines of force to stretch radially outwards for long distances, producing the unique "magnetodisc". A complex buffer zone exits between the outer edge of this disc and the outer boundary of the magnetosphere, which separates the magnetized Jovian atmosphere from solar wind plasma. Upstream of the magnetosphere, a detached bow shock forms that slows the solar wind and allows it to be deflected around the magnetosphere.

The trajectory past Jupiter of Ulysses, the fifth spacecraft to fly through the Jovian magnetosphere, where the open circle represents the point of closest approach at 6.3 Jovian radii $\left(R_{J}\right)$. Unique aspects of the flight path are: a) penetration of the 10 plasma torus, in basically a N-S direction: b) the spacecraft radio signal passed through the torus for a significant time allowing the electron density to be probed; c) the outbound passage was through the magnetosphere's previously unexplored dusk sector.

and ions. A surprising finding, with signatures in the data from many of the instruments, was that even close into the planet $\left(\approx 9 R_{\mathrm{J}}\right)$, Ulysses apparently made an excursion out of the radiation belts at magnetic latitudes of only $48^{\circ}$. The exact nature of the high-latitude environment outside the radiation belts is still a matter of discussion, but features characteristic of solar wind conditions were observed. For example, the energetic particle instruments registered counting rates close to interplanetary background levels.

Jupiter is a prolific source of natural radio waves, emitting at many wavelengths. The unique direction finding capability and high sensitivity of the Ulysses radio and plasma wave experiment have provided new insights and clues as to the origin of these radio signals. For example, a characteristic electromagnetic emission called narrowband kilometric radiation, has been found to originate from five discrete sources that are located in the outer regions of the lo plasma torus, and which co-rotate with the planet. Ulysses observations of the hectometric radiation revealed narrow, latitudinal beaming along the magnetic equator, and provided additional constraints on existing models for the source of this radio emission. Bursts of radio emission showing a characteristic rapid drift in frequency, so-called "Jovian Type III" events, have been studied in detail and appear to be a major component of Jupiter's radio spectrum.
A major discovery during the outbound pass was the existence at high latitudes of very strong counterflowing streams of electrons and ions, constituting large currents that apparently feed into the auroral regions. Further study of this effect will hopefully reveal if these currents are sufficient to power the Jovian aurora. In the same regions, periodic bursts of $\mathrm{MeV}$ electrons were observed flowing away from the planet. Preliminary estimates indicate that these bursts may represent a significant fraction of the population of relativistic electrons found in interplanetary space. Data from many of the Ulysses experiments point to the fact that the dusk-side magnetosphere, where the fields and plasmas rotate from the compressed day side into the magnetotail, is highly dynamic.

Papers summarising the initial results from the Jupiter fly-by appear in Science 257 (11 September 1992). All experiments were conceived, designed and built, and the data reduced, interpreted and analysed, by collaborations responsible for each instrument, with European participation mainly financed by institutions or national funds. Aside from detailed examination of existing data, the next major phase of the Ulysses adventure will be the much more prolonged flights across the sun's poles.

K.-P. Wenzel and R.G. Marsden ESTEC, Noordwijk 